\title{
SOROPREVALÊNCIA E VARIÁVEIS EPIDEMIOLÓGICAS ASSOCIADAS À LEISHMANIOSE VISCERAL CANINA EM ÁREA ENDÊMICA NO MUNICÍPIO DE SÃO LUÍS, MARANHÃO, BRASIL
}

\author{
David Soeiro Barbosa, ${ }^{1}$ Alessandra Lima Rocha, ${ }^{2}$ Andressa Almeida Santana, ${ }^{3}$ Celeste da Silva Freitas de \\ Souza, ${ }^{4}$ Ricardo Augusto Dias, ${ }^{5}$ Lívio Martins Costa-Júnior ${ }^{6}$ e Ana Lúcia Abreu-Silva ${ }^{7}$ \\ 1. Pós-graduando da Escola Nacional de Saúde Pública/Fiocruz. E-mail: davidsoeiro@gmail.com \\ 2. Graduanda do Curso de Medicina Veterinária da UEMA \\ 3. Pós-graduanda da Rede Nordeste de Biotecnologia - Renorbio \\ 4. Tecnologista em Saúde Pública da Fundação Oswaldo Cruz \\ 5. Professor doutor da Faculdade de Medicina Veterinária e Zootecnia/USP \\ 6. Professor doutor do Centro de Ciências Agrárias e Ambientais/UFMA \\ 7. Professora doutora do Centro de Ciências Agrárias/UEMA.
}

\section{RESUMO}

Este trabalho teve como objetivos determinar a soroprevalência e as variáveis epidemiológicas associadas com a infecção de Leishmania spp. em cães de cinco localidades no Distrito do Tirirical no município de São Luís, Maranhão. Foram visitadas 72 moradias, perfazendo uma amostra de cem cães domiciliados, e aplicados questionários com o objetivo de determinar os fatores que poderiam estar relacionados com a ocorrência da infecção. Utilizaram-se como variáveis: proximidade da moradia com a mata, existência de criação/abrigo de animais de produção e de animais silvestres, sexo, idade, raça, além de exame clínico do animal, com observação da presença de sinais clínicos compatíveis com a doença. A análise sorológica demonstrou que 67 amostras apresentaram-se positivas para Leishmania spp. Os sinais clínicos observados foram linfadenopatia localizada, alopecia, pelo opaco, emagrecimento, úlceras cutâneas, descamação furfurácea, ceratoconjuntivite, e onicogrifose. Animais das localidades Cruzeiro de Santa Bárbara e Cajupari, ambas localizadas próximas de matas, têm 3,4 e 12,0 vezes mais chances de serem soropositivos para Leishmania spp. do que aqueles das outras localidades estudadas. Não se verificou correlação entre as outras variáveis estudadas e soropositividade para Leishmania spp.

PALAVRAS-CHAVES: Epidemiologia, fatores associados, leishmaniose visceral canina, soroprevalência.

\section{SEROPREVALENCE AND ASSOCIATED EPIDEMIOLOGIC VARIABLES WITH CANINE VISCERAL LEISHMANIASIS IN ENDEMIC AREA, SÃO LUIS, MARANHÃO STATE, BRAZIL}

This work aimed to determine the seroprevalence and epidemiological variables associated to Leishmania spp. infection in dogs from five locations of Tirirical District, in São Luís, Maranhão State, Brazil. Seventy-two houses were visited and samples of one hundred dogs were taken. A questionnaire was applied in order to investigate the possible factors related to the Leishmania infection. The following variables were used: forest proximity from the residence, livestock of domestic animals or shelter of wild animals, sex, age and breed. Clinical examination was also performed looking for clinical signs compatible to the infection. The serological analysis revealed that 67 samples were reactive. The clinical signs observed were focal lymphadenophaty, alopecia, weight loss, cutaneous ulcers, skin desquamation, keratoconjuntivitis and onycogrifosis. Animals from Cruzeiro de Santa Bárbara and Cajupari, both locations near the forest, have 3.4 and 12.0 more chances of being reactive to Leishmania spp. than the ones in the other studied locations. No statistical relation was found between seropositive dogs and any other variables.

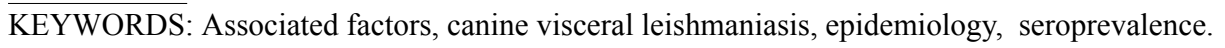




\section{INTRODUÇÃO}

As leishmanioses são doenças causadas por protozoários tripanosomatídeos do gênero Leishmania, consideradas primariamente como zoonoses. Constituem-se importantes problemas de saúde pública, encontrando-se atualmente entre as doenças tropicais prioritárias da Organização Mundial de Saúde (MINISTÉRIO DA SAÚDE, 2006). A leishmaniose visceral (LV) tem como agente etiológico, no Novo Mundo, a espécie Leishmania (Leishmania) chagasi, sendo Lutzomyia longipalpis o principal transmissor da doença no Brasil (MENDES et al., 2002). É uma doença de notificação compulsória, que apresenta taxas elevadas de incidência e letalidade, sendo endêmica em 62 países, com um total estimado de 200 milhões de pessoas sob risco de infecção. Sua distribuição ocorre na Ásia, na Europa, no Oriente Médio, na África e nas Américas. Na América Latina a doença já foi descrita em pelo menos doze países, com a maioria dos casos provenientes do Brasil (GONTIJO \& MELO, 2004; MINISTÉRIO DA SAÚDE, 2006).

No Brasil, a doença apresenta ampla distribuição territorial, com aspectos geográficos, climáticos e sociais diferenciados. Atualmente vem se expandindo para áreas urbanas de médio e grande porte, mudando seu perfil epidemiológico, antes eminentemente rural. Sua distribuição geográfica abrange as regiões CentroOeste, Sudeste, Norte e Nordeste, sendo esta última a que apresenta a maioria dos casos notificados no país (MINISTÉRIO DA SAÚDE, 2006). Estudos têm relatado a ocorrência de casos de LV canina na Região Sul do país (POCAI et al., 1998; KRAUSPENHAR et al., 2007; THOMAZ-SOCCOL et al., 2009). O estabelecimento da LV como epidemia no Maranhão ocorreu a partir de 1982, na cidade de São Luís, em uma extensão do Bairro do Tirirical, com registro de 1.089 casos em quinze anos, período em que recebeu grande fluxo migratório oriundo em parte do deslocamento de lavradores de Estados vizinhos, em virtude do êxodo de famílias que viviam na zona rural do Maranhão (MENDES et al., 2002). Posteriormente houve expansão para outras áreas da Ilha de São Luís, como São José de Ribamar e Paço do Lumiar. A partir de 1988, a doença passa por uma segunda fase da epidemia, com franca expansão para áreas suburbanas da Ilha de São Luís, período em que o Maranhão transformou-se em um dos Estados com áreas de maior problema em relação à doença (COSTA et al., 1995).

A urbanização da LV no Brasil nas últimas décadas tem criado condições epidemiológicas favoráveis para a manutenção da doença, com densa população de reservatórios, vetores e humanos, distribuídos no mesmo ambiente tropical que é extremamente favorável à transmissão (DINIZ et al., 2008). Os bairros com animais positivos, em geral, estão localizados próximos de pequenas áreas de mata característica da região, o que viabilizaria a presença do vetor junto às residências (MARZOCHI, 1994; MOURA et al., 1999). O controle efetivo dessa doença requer a diminuição de sua incidência em cães, que servem como reservatório para a doença no homem. Porém há discordância entre os pesquisadores de que a LV canina seja a causa necessária para a infecção humana, apesar de os estudos apontarem nessa direção (GONTIJO \& MELO, 2004).

Em virtude da importância epidemiológica do cão como reservatório da leishmaniose visceral e da situação endêmica da doença no Estado do Maranhão, além da constante busca por alternativas concretas e viáveis, tanto no campo do diagnóstico e prognóstico quanto da imunoprofilaxia da LV canina, propostas inovadoras e interdisciplinares visando o desenvolvimento de conhecimentos básicos e de alternativas para o controle da leishmaniose devem ser priorizadas. Torna-se importante determinar seu perfil epidemiológico, assim como os fatores que implicam a ocorrência da doença. Dessa forma, este trabalho teve como objetivos determinar a soroprevalência e as variáveis epidemiológicas associados com a infecção de Leishmania spp. em cães residentes no Distrito do Tirirical de São Luís, MA.

\section{MATERIAL E MÉTODOS}

Área de estudo

A área de estudo foi o município de São Luís, localizado na região norte do Estado do Maranhão (Figura 1), com área de $827 \mathrm{~km}^{2}, 24$ metros de altitude, e como coordenadas geográficas $2^{\circ} 31^{\prime} \mathrm{S}$ e $44^{\circ} 18^{\prime} \quad 0$. O clima é do tipo tropical, quente e semiúmido da Zona Equatorial. São Luís tem duas estações distintas: o verão, de julho a dezembro, e o inverno, de janeiro a 
junho. O verão é quente e seco com ventos frescos e o inverno é chuvoso. Possui média pluviométrica de $1953 \mathrm{~mm}$.

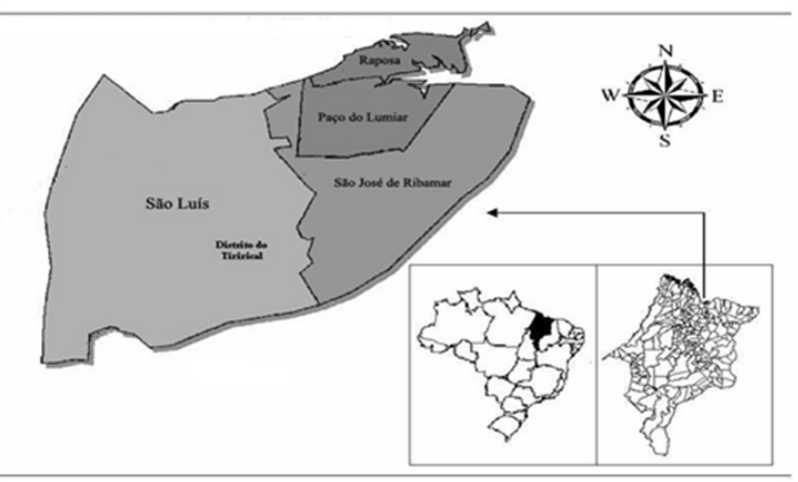

FIGURA 1. Distrito do Tirirical, São Luís, Maranhão, Brasil. (FONTES: IBGE e SEPLAN/ MA.

O município divide-se em sete distritos sanitários: Itaqui-Bacanga, Centro, Tirirical, Vila Esperança, Cohab, Bequimão e Coroadinho. O presente estudo foi realizado em cinco localidades, incluindo bairros, vilas e povoados que fazem parte do Distrito do Tirirical. A escolha foi baseada nos seguintes fatores: positividade canina para a doença, localização em área com características urbanas, semiurbanas e rurais e elevado índice de casos humanos. As localidades estudadas foram a Cidade Operária (ambiente com características urbanas); Cruzeiro de Santa Bárbara, Residencial José Reinaldo Tavares e Recanto Canaã (ambiente com características rurais e urbanas); Cajupari (ambiente com características rurais). Também foi levado em consideração, na escolha das localidades do estudo, o número de casos humanos e caninos notificados pelo Centro de Controle de Zoonoses de São Luís, MA. As escolhas recaíram sobre o Residencial José Reinaldo Tavares, pelo fato de ser a maior localidade do distrito do Tirirical com menor número de casos caninos, e sobre o Recanto Canaã, porque, dentre as localidades, é a que apresenta maior número de casos humanos.

Amostras e diagnóstico laboratorial

Para diagnóstico da infecção canina, foram obtidas amostras de cem animais domiciliados, sintomáticos e assintomáticos, de 72 moradias visitadas, com a seguinte distribuição por localidade: Cidade Operária (21); Cru- zeiro de Santa Bárbara (21), Residencial José Reinaldo Tavares (23); Recanto Canaã (19) e Cajupari (16). O número de animais incluídos no estudo por localidade foi resultante dos moradores que quiseram colaborar com a pesquisa, cujo somatório levou à amostra final. As localidades com menor número de amostras, caso do Cajupari e Recanto Canaã, são as que possuem menor número de casas com criação de cães, o que é uma limitação deste estudo.

Submeteram-se os animais a exames físicos para observação de sinais clínicos ou não da leishmaniose visceral canina. Em seguida foram coletadas amostras de sangue por meio de punção da veia cefálica, para realização da Reação de Imunofluorescência Indireta (RIFI) (BIOMANGUINHOS/FIOCRUZ), para detecção de anticorpos contra Leishmania spp. em soros de cão. Consideraram-se positivas as amostras com titulação 1/40, conforme preconizado pelo Ministério da Saúde. Os ensaios foram realizados nos Laboratórios de Anatomopatologia e Virologia do curso de Medicina Veterinária da Universidade Estadual do Maranhão, Campus Paulo VI.

Ficha de investigação

Foram preenchidas fichas de investigação, junto aos moradores, com o objetivo de determinar as variáveis epidemiológicas que poderiam estar correlacionados com a ocorrência da doença, como proximidade da moradia com a mata; existência de criação/abrigo de animais de produção e animais silvestres; além de características do animal, como sexo, idade, raça, tamanho e cor do pelo.

Análise estatística

Realizou-se a associação entre os resultados da sorologia e as variáveis pelo qui-quadrado, fazendo uso do programa BioEstat 3.0. A regressão logística múltipla foi feita com as variáveis estatisticamente significativas observadas na análise univariada, mediante o programa Minitab 14.

\section{RESULTADOS E DISCUSSÃO}

A análise sorológica demonstrou que, das cem amostras analisadas, 67 (67\%) apresentaram-se positivas para Leishmania spp. 
Na determinação da soroprevalência por localidade, constatou-se que Cajupari e Cruzeiro de Santa Bárbara apresentaram elevados índices de positividade, com $94 \%$ e $81 \%$, respectivamente. O Residencial José Reinaldo Tavares mostrou a soroprevalência mais baixa (48\%). Por fim, nas localidades Recanto Canaã e Cidade Operária, observaram-se soroprevalências de $53 \%$ e $67 \%$, respectivamente.

As localidades mostraram características diversas. Cajupari e Cruzeiro de Santa Bárbara localizam-se próximos de matas, com relatos de presença de animais silvestres nas imediações do domicílio. No Residencial José Reinaldo Tavares, as residências ficam próximas da mata e 65,2\% delas não possuem abrigo e criação de animais. Também não foi relatada, pelos moradores, a presença de animais silvestres nas proximidades dos domicílios. Na localidade Recanto Canaã, as residências são próximas da mata e a maioria dos moradores da localidade relatou presença de animais silvestres nas imediações dos domicílios, tanto de gambás, também conhecidos como mucuras, na região (Didelphis marsupialis), quanto de raposas (Cerdocyon thous). A Cidade Operária é uma área urbanizada, sem proximidade com a mata, não possui criação de animais no domicílio e com poucos relatos de animais silvestres observados nas suas imediações. Tais descrições foram importantes para caracterizar o perfil da doença nas localidades, relacionando-a com as variáveis epidemiológicas, buscando identificar áreas de maior risco para a ocorrência da leishmaniose canina.

A soroprevalência elevada encontrada na Cidade Operária (67\%) pode ser justificada pela expansão que a leishmaniose visceral tem demonstrado no ambiente urbano nas últimas décadas, o que vem sendo observado no município de São Luís, MA (MINISTÉRIO DA SAÚDE, 2006; DINIZ et al., 2008).

Neste estudo, as localidades Cajupari e Cruzeiro de Santa Bárbara apresentaram os maiores índices de positividade, com $94 \%$ e $81 \%$, respectivamente. Ambas são localizadas próximas de matas e há relatos da presença de animais silvestres nas imediações do domicílio. Alguns estudos relatam que, em localidades onde há presença de animais silvestres nas proximidades dos domicílios e em habitações situadas perto de matas em áreas de ocupação recente e com condições sanitárias insatisfatórias, existe uma tendência para abundância de vetores e reservatórios, o que contribui para a manutenção e disseminação da doença, revelando alta soroprevalência canina (GUIMARÃES et al., 2005; OLIVEIRA, et al., 2006).

A população de cães positivos analisada apresentou maior número de animais assintomáticos $(58,2 \%)$, o que corrobora o que é descrito na literatura (OLIVEIRA et al., 2001; MADEIRA et al., 2004; ALMEIDA et al., 2009). Dentre os sinais clínicos observados nos animais positivos, a linfadenopatia localizada (linfonodo poplíteo) foi o mais frequente, acometendo 27 cães (96,4\%). Outros sinais encontrados foram úlceras cutâneas $(60,7 \%)$, onicogrifose $(50 \%)$, pelo opaco $(25 \%)$, ceratoconjuntivite $(21,4 \%)$, eczema furfuráceo $(21,4 \%)$, alopecia $(14,2 \%)$ e emagrecimento $(10,7 \%)$. Não foram observados animais com formações nodulares. Em estudos realizados em Recife, PE, os sinais clínicos mais frequentes foram úlceras cutâneas, seguidas de linfadenopatia, emagrecimento, onicogrifose e oftalmopatias (ALBUQUERQUE et al., 2007).

Neste estudo, constatou-se que animais que vivem nas localidades Cruzeiro de Santa Bárbara e Cajupari, ambas localizadas próximas de matas, têm 3,4 $(p=0,04)$ e $12,0(p=0,02)$ vezes maior probabilidade de serem soropositivos para Leishmania spp. do que aqueles que vivem nas outras localidades estudadas. Desse modo, estratégias de vigilância epidemiológica e controle da doença devem ser buscadas, visando estabelecer ações específicas direcionadas para essas áreas, onde o risco de ocorrência é maior. Isso, no entanto, não significa deixar de realizar as ações nas outras áreas, considerando-se que se trata de um área endêmica para LVC. Também é recomendada a realização de estudos para melhor compreender o ciclo silvestre da doença e assim propor soluções mais efetivas no controle da doença. As áreas urbanas, como observado na localidade Cidade Operária, também sofrem, atualmente, um incremento na casuística de leishmaniose. O processo de urbanização da doença vem se expandindo, inclusive, para áreas densamente povoadas. O grande número de vetores e reservatórios é extremamente favorável para a ocorrência da doença, devendo também ser controlada, evitando o risco para a saúde humana e animal (DINIZ et al., 2008).

Não se verificou correlação entre as outras variáveis estudadas e soropositividade para Leishmania spp (Tabela 1). Em trabalhos realizados em Mossoró, $\mathrm{RN}$, não foi evidenciada diferença estatisticamente 
significativa na positividade dos cães entre o meio rural e urbano. Entre os animais peridomiciliados, houve maior soroprevalência tanto na área rural quanto urbana, e as fêmeas da zona rural foram mais prevalentes (AMÓRA et al., 2006). Nesta pesquisa, notou-se que as áreas próximas de matas apresentaram soroprevalência mais elevada. Não foi evidenciada predisposição sexual.

TABELA 1. Taxa de prevalência e variáveis epidemiológicas associadas à soropositividade canina para Leishmania spp. no distrito do Tirirical, São Luís, Maranhão

\begin{tabular}{|c|c|c|c|c|}
\hline Variáveis & Prevalência $+/$ n (\%) & OR & $\mathrm{P}$ & IC 95\% \\
\hline \multicolumn{5}{|l|}{ Classificação clínica } \\
\hline Sintomático & $28 / 39(71,7 \%)$ & \multirow{2}{*}{1,4} & \multirow{2}{*}{0,55} & \multirow{2}{*}{$0,60-3,43$} \\
\hline Assintomático & $39 / 61(63,9 \%)$ & & & \\
\hline \multicolumn{5}{|l|}{$\mathrm{Sexo}^{\mathrm{a}}$} \\
\hline Fêmea & $37 / 49(75,5 \%)$ & \multirow{2}{*}{2,2} & \multirow{2}{*}{0,08} & \multirow{2}{*}{$0,89-5,45$} \\
\hline Macho & $30 / 51(58,8 \%)$ & & & \\
\hline \multicolumn{5}{|l|}{ Raça } \\
\hline Não & $59 / 87(67,8 \%)$ & \multirow{2}{*}{1,3} & \multirow{2}{*}{0,89} & \multirow{2}{*}{$0,39-4,39$} \\
\hline $\operatorname{Sim}$ & $8 / 13(61,5 \%)$ & & & \\
\hline \multicolumn{5}{|l|}{ Idade } \\
\hline Até 2 anos & $26 / 42(66,6 \%)$ & \multirow{2}{*}{0,6} & \multirow{2}{*}{0,48} & \multirow{2}{*}{$0,29-1,56$} \\
\hline$>2$ anos & $41 / 58(70,6 \%)$ & & & \\
\hline \multicolumn{5}{|l|}{ Tamanho do pelo } \\
\hline Curto & $47 / 72(65,2 \%)$ & \multirow{2}{*}{0,7} & \multirow{2}{*}{0,72} & \multirow[b]{2}{*}{$0,29-1,95$} \\
\hline Longo & $20 / 28(71,4 \%)$ & & & \\
\hline \multicolumn{5}{|l|}{ Cor de pelo } \\
\hline Claro & $41 / 58(70,6 \%)$ & \multirow{2}{*}{1,4} & \multirow{2}{*}{0,48} & \\
\hline Escuro & $26 / 42(61,9 \%)$ & & & $0,64-3,44$ \\
\hline Localidade & & & & \\
\hline J. R. Tavares ${ }^{\text {a }}$ & $11 / 23(47,8 \%)$ & & & \\
\hline Outras localidades & $56 / 77(72,7 \%)$ & 0,5 & 0,27 & $0,16-1,73$ \\
\hline Cidade Operária & $14 / 21(66,6 \%)$ & & 082 & \\
\hline Outras localidades & $53 / 79(67 \%)$ & 1,0 & 0,82 & $0,36-2,38$ \\
\hline C. S. Bárbara ${ }^{a}$ & $17 / 21(80,9 \%)$ & & & \\
\hline Outras localidades & $50 / 79(63,2 \%)$ & 3,4 & $0,04^{*}$ & $1,03-11,26$ \\
\hline Recanto Canaã & $10 / 19(52,6 \%)$ & & & \\
\hline Outras localidades & $57 / 81(70,3 \%)$ & 2,1 & 0,22 & $0,77-5,92$ \\
\hline Cajupari $^{\mathrm{a}}$ & $15 / 16(93,7 \%)$ & 120 & & \\
\hline Outras localidades & $52 / 84(61,9 \%)$ & 12,0 & $0,01^{\top}$ & $1,49-96,4 /$ \\
\hline Proximidade da mata ${ }^{a}$ & & & & \\
\hline Sim & $42 / 56(75 \%)$ & & & \\
\hline Não & $25 / 44(56,8 \%)$ & 0,36 & 0,18 & $0,08-1,62$ \\
\hline Forma de criação & & & & \\
\hline Solto & $41 / 57(71,9 \%)$ & & & \\
\hline Preso & $26 / 43(60,4 \%)$ & 1,7 & 0,29 & $0,74-3,97$ \\
\hline Local que cria & & & & \\
\hline Domicílio & $5 / 8(62,5 \%)$ & & & \\
\hline Peridomicílio & $62 / 92(67,3 \%)$ & 0,8 & 0,91 & $0,18-3,6$ \\
\hline Criação de animais & & & & \\
\hline Sim & $32 / 46(69,5 \%)$ & & & \\
\hline Não & $35 / 54(64,8 \%)$ & 1,2 & 0,77 & $0,53-2,87$ \\
\hline Animais silvestres ${ }^{a}$ & & & & \\
\hline Sim & $38 / 51(74,5 \%)$ & & & \\
\hline Não & $29 / 49(59,1 \%)$ & 0,57 & 0,48 & $0,12-2,71$ \\
\hline Abrigo de animais & & & & \\
\hline Sim & $32 / 45(71,1 \%)$ & 13 & & \\
\hline Não & $35 / 55(63,6 \%)$ & 1,3 & 0,61 & $0,58-3,18$ \\
\hline
\end{tabular}

${ }^{*}=$ Resultados estatisticamente significativos $(\mathrm{P}>0,05) \quad /{ }^{\mathrm{a}}=$ Variáveis utilizadas na análise múltipla $\quad / \quad{ }^{\mathrm{n}}=$ Número de amostras variável 
Estudos realizados descreveram que animais de pelo curto têm maiores riscos de serem infectados por Leishmania. A criação de porcos e galinhas aumenta o risco para a ocorrência da doença. Também sugerem que o risco aumenta após o primeiro ano de vida do animal, permanecendo estável após este. Sexo, raça, grau de confinamento não foram fatores significativos para incidência da doença (MOREIRA et al., 2003). No presente estudo não se observou correlação entre a positividade canina com o tamanho do pelo e a criação de outros animais. Contudo, os resultados aqui apresentados estão de acordo quanto à ausência de predisposição para a infecção relacionada ao sexo, idade, local e forma de criação do animal.

A leishmaniose visceral é atualmente um grave problema de saúde pública, apresentando ainda entraves para sua vigilância e controle, como falta de padronização dos métodos de diagnóstico da infecção humana e canina, discordância entre os estudos que avaliam o impacto da eliminação de cães soropositivos na prevalência da infecção humana, demonstração de que outros reservatórios podem ser fonte de infecção da L. chagasi, como os canídeos silvestres e os marsupiais, e a escassez de estudos sobre o impacto das ações de controle dirigidas contra os vetores (GONTIJO \& MELO, 2004). A ocorrência da doença envolve aspectos biológicos, ambientais e sociais. Uma vez que a destruição das matas pelo homem vem causando um desequilíbrio, ocorre um aumento do número de casos, favorecendo, assim, a expansão e a urbanização da doença. Apesar do processo de urbanização, constata-se que são as localidades onde as residências têm proximidade com a mata que apresentaram maior número de animais acometidos pela infecção.

\section{CONCLUSÕES}

A soroprevalência da LVC é elevada nas localidades estudadas. As localidades Cajupari e Cruzeiro de Santa Bárbara apresentaram os maiores índices de positividade canina. O Residencial José Reinaldo Tavares mostrou a menor soroprevalência. Animais que vivem nas localidades Cruzeiro de Santa Bárbara e Cajupari têm maior probabilidade de serem soropositivos para Leishmania spp. do que animais que vivem nas outras localidades estudadas. Com exceção da localidade, não se encontrou correlação entre as outras variáveis estudadas e soropositividade para Leishmania spp.

\section{AGRADECIMENTOS}

À Universidade Estadual do Maranhão, pela concessão de bolsa de Iniciação Científica (programa BIC/UEMA), no período de novembro de 2006 a novembro de 2007. À FAPEMA, pela concessão de Bolsa de Apoio Técnico no período de fevereiro de 2008 a fevereiro de 2009.

\section{REFERÊNCIAS}

ALMEIDA, A. B. P. F.; FARIA, R. P.; PIMENTEL, M. F. A.; DAHROUG, M. A. A.; TURBINO, N. C. M. R.; SOUSA, V. R. $\mathrm{F}$. Inquérito soroepidemiológico de leishmaniose canina em áreas endêmicas de Cuiabá, Estado de Mato Grosso. Revista da Sociedade Brasileira de Medicina Tropical, v. 42, n. 2, 2009.

AMORA, S. S. A.; SANTOS, M. J. P.; ALVES, N. D.; COSTA, S. C. G.; CALABRESE, K. S.; MONTEIRO, A. J.; ROCHA, M. F. G. Fatores relacionados com a positividade de cães para Leishmaniose visceral em área endêmica do Estado do Rio Grande do Norte, Brasil. Ciência Rural, v. 36, p. 1854-1859, 2006.

ALBUQUERQUE, A. R.; ARAGÃO, F. R; FAUSTINO, M. A. G.; GOMES, Y. M.; LIRA, R. A.; NAKASAWA, M.; ALVES, L. C. Aspectos clínicos de cães naturalmente infectados por Leishmania (Leishmania) chagasi na região metropolitana do Recife. Clínica Veterinária, Ano XII, n. 71, 2007.

COSTA, J. M. L.; VIANA, G. M. C.; SALDANHA, A. C. R.; NASCIMENTO, M. D. S. B.; ALVIM, A. C.; BURATTINI, M. N.; SILVA; A. R. Leishmaniose visceral no Estado do Maranhão: a evolução de uma epidemia. Cadernos de Saúde Pública, v. 11 p. 321-324, 1995.

DINIZ, S. A.; SILVA F. L.; CARVALHO-NETA, A. V.; BUENO, R.; GUERRA, R. M. S. N. C.; ABREU-SILVA, A. L.; SANTOS, R. L. Animal reservoirs for visceral leishmaniasis in densely populated urban áreas. Journal of Infection in Developing Countries, v. 2, n. 1, p. 24-33, 2008.

GONTIJO, C. M. F.; MELO, M. N. Leishmaniose visceral no Brasil: quadro atual, desafios e perspectivas. Revista Brasileira de Epidemiologia, v. 7, n. 3, p. 338-349, 2004.

GUIMARÃES, K. S.; BATISTA, Z. S.; DIAS, E. L.; GUERRA; R. M. N. C.; COSTA, A. D. C. C.; OLIVEIRA, A. S.; CALABRESE; K. S.; CARDOSO; F. O.; SOUZA, C. S. F.; ZAVERUCHA DO 
VALE T.; GONÇALVES DA COSTA, S. C.; ABREU-SILVA, A. L. Canine visceral leishmaniasis in São José de Ribamar, Maranhão State, Brazil. Veterinary Parasitology, v. 131, p. 305-309, 2005.

KRAUSPENHAR, C.; BECK, C.; SPEROTTO, V.; SILVA, A. A.; BASTOS, R.; RODRIGUES, L. Leishmaniose visceral em um canino de Cruz Alta, Rio Grande do Sul, Brasil. Ciência Rural, v. $37,2007$.

MADEIRA, M. F.; SCHUBACH, A. O.; SCHUBACH, T. M. P.; LEAL, C. A.; MARZOCHI, M. C. A. Identification of Leishmania (Leishmania) chagasi isolated from healthy skin of symptomatic and asymptomatic dogs seropositive for Leishmaniasis in the municipality of Rio de Janeiro, Brazil. Brazilian Journal Infectious Diseases, v.8, p.440-444, 2004.

MARZOCHI, M. C. A. Epidemiologia das leishmanioses no Brasil. Revista de Patologia Tropical, v. 23, n. 2, p. 82-84, 1994.

MENDES, S. W.; SILVA, A. A. M.; TROVÂO, J. R.; SILVA, A. R.; COSTA J. M. L. Expansão espacial da Leishmaniose visceral Americana em São Luís do Maranhão, Brasil. Revista da Sociedade Brasileira de Medicina Tropical, v. 35, p. 227-231, 2002.

MINISTÉRIO DA SAÚDE DO BRASIL. Secretaria de Vigilância em Saúde. Departamento de Vigilância Epidemiológica. Manual de vigilância e controle da Leishmaniose visceral. Brasília, DF; 2006. (Série A. Normas e Manuais Técnicos).

MOREIRA, J. R.; DUARTE E.; SOUZA, V. M. M.; SREENIVASAN, M.; LOPES, N. L.; SILVA, R. B. B.; CARVALHO, L. P. Pe- ridomestic risk factors for canine Leishmaniasis in urban dwelling: new findings from a prospective study in Brazil. American Journal of Tropical Medicine and Hygiene, v. 69, p. 393-397, 2003.

MOURA, S. T.; FERNANDES, G. C. N; PANDOLPHO, V. C.; RODRIGUES E SILVA, R. Diagnosis of canine leishmaniasis in the urban area of the District of Cuiabá, State of Mato Grosso, Brazil. Brazilian Journal of Veterinary Research and Animal Science, v. 36 n. 2, 1999.

OLIVEIRA, A. C.; ABREU-SILVA, A. L.; LIMA, T. B.; SILVA, A. P. C.; REIS, L. F.; BARBOSA, D. S.; GUERRA, R. M. S. N. C. Soroprevalência da leishmaniose visceral canina no bairro Jardim São Raimundo em São Luís. Revista da Sociedade Brasileira de Medicina Tropical, Suppl. v. 1, p. 140-143, 2006.

OLIVEIRA, C. D. L.; ASSUNÇÃO, R. M.; REIS, I. A.; PROIETTI, F. A. Spatial distribution of human and canine visceral leishmaniasis in Belo Horizonte, Minas Gerais State, Brasil, 1994-1997. Cadernos de Saúde Pública, v. 17, p. 1231-1239, 2001.

POCAI, E. A.; FROZZA, L.; HEADLEY, S. A.; GRAÇA, D. L. Leishmaniose visceral (calazar): cinco casos em cães de Santa Maria, Rio Grande do Sul, Brasil. Ciência Rural, v. 28, p. 501505, 1998.

THOMAZ-SOCCOL, V.; CASTRO, E. A.; NAVARRO, I. T.; FARIAS, M. R.; SOUZA, L. M.; CARVALHO; Y.; BISPO, S.; MEMBRIVE, N. A.; MIZZONO, J. C.; TRUPPEL, J.; BUENO, W.; LUZ, E. Casos alóctones de leishmaniose visceral canina no Paraná, Brasil: implicações epidemiológicas. Revista Brasileira de Parasitologia Veterinária, v. 18, n. 3, p. 46-51, jul.-set. 2009.

Protocolado em: 9 abr. 2009. Aceito em: 17 mar. 2010. 